\title{
Drug screening approach combines epigenetic sensitization with immunochemotherapy in cancer
}

\author{
Chiara Facciotto ${ }^{1 \dagger}$, Julia Casado ${ }^{1 \dagger}$, Laura Turunen², Suvi-Katri Leivonen ${ }^{3,4}$, Manuela Tumiati', Ville Rantanen ${ }^{1}$, \\ Liisa Kauppi ${ }^{1}$, Rainer Lehtonen ${ }^{1}$, Sirpa Leppä ${ }^{3,4}$, Krister Wennerberg $^{2}$ and Sampsa Hautaniemi ${ }^{1^{*}}$ (D)
}

\begin{abstract}
Background: The epigenome plays a key role in cancer heterogeneity and drug resistance. Hence, a number of epigenetic inhibitors have been developed and tested in cancers. The major focus of most studies so far has been on the cytotoxic effect of these compounds, and only few have investigated the ability to revert the resistant phenotype in cancer cells. Hence, there is a need for a systematic methodology to unravel the mechanisms behind epigenetic sensitization.

Results: We have developed a high-throughput protocol to screen non-simultaneous drug combinations, and used it to investigate the reprogramming potential of epigenetic inhibitors. We demonstrated the effectiveness of our protocol by screening 60 epigenetic compounds on diffuse large B-cell lymphoma (DLBCL) cells. We identified several histone deacetylase (HDAC) and histone methyltransferase (HMT) inhibitors that acted synergistically with doxorubicin and rituximab. These two classes of epigenetic inhibitors achieved sensitization by disrupting DNA repair, cell cycle, and apoptotic signaling. The data used to perform these analyses are easily browsable through our Results Explorer. Additionally, we showed that these inhibitors achieve sensitization at lower doses than those required to induce cytotoxicity.

Conclusions: Our drug screening approach provides a systematic framework to test non-simultaneous drug combinations. This methodology identified HDAC and HMT inhibitors as successful sensitizing compounds in treatment-resistant DLBCL. Further investigation into the mechanisms behind successful epigenetic sensitization highlighted DNA repair, cell cycle, and apoptosis as the most dysregulated pathways. Altogether, our method adds supporting evidence in the use of epigenetic inhibitors as sensitizing agents in clinical settings.
\end{abstract}

Keywords: Epigenetic reprogramming, High-throughput drug screening, epigenetic inhibitors, Drug resistance in cancer, DLBCL

\section{Background}

DNA methylation and histone modifications dynamically regulate the chromatin structure, playing an important role in defining and maintaining cells' identity [1]. Epigenome disruption has also been linked to several diseases including cancer [2-4] and causing treatment failure [5]. Inhibitors of enzymes responsible for writing, reading, and erasing epigenetic marks can be used as cytotoxic

\footnotetext{
* Correspondence: sampsa.hautaniemi@helsinki.fi

${ }^{+}$Chiara Facciotto and Julia Casado contributed equally to this work.

${ }^{1}$ Research Program in Systems Oncology, Faculty of Medicine, University of Helsinki, PO Box 63, Helsinki, Finland

Full list of author information is available at the end of the article
}

agents on tumor cells and several of them are already in clinical trial [6]. Importantly, epigenetic compounds have recently been shown to reprogram cellular phenotypes, which enable a novel treatment approach that exploits the plastic nature of the epigenome to turn drug resistant cancer cells into sensitive ones [7, 8].

Episensitization studies so far have focused on manually testing the sensitizing potential of one or few epigenetic compounds $[7,9]$. To advance the episensitization experiments to larger-scale level, we developed a novel, high-throughput drug screening protocol to enable non-simultaneous administration of multiple compounds over a period spanning

(c) The Author(s). 2019 Open Access This article is distributed under the terms of the Creative Commons Attribution 4.0 International License (http://creativecommons.org/licenses/by/4.0/), which permits unrestricted use, distribution, and reproduction in any medium, provided you give appropriate credit to the original author(s) and the source, provide a link to the Creative Commons license, and indicate if changes were made. The Creative Commons Public Domain Dedication waiver (http://creativecommons.org/publicdomain/zero/1.0/) applies to the data made available in this article, unless otherwise stated. 
several days. We also created an inhibitor collection to test all main classes of epigenetic enzymes, comprising DNA methyltransferases (DNMTs), histone methyltransferases (HMTs), histone acetyltransferases (HATs), histone demethylases (HDMs), histone deacetylases (HDACs), and bromodomains (BRDs).

We systematically assessed the ability of epigenetic inhibitors to overcome treatment resistance in diffuse large B-cell lymphoma (DLBCL). The standard of care therapy given to previously untreated DLBCL patients of all ages and subtypes is the immunochemotherapy called RCHOP [10]. This combination consists of rituximab, cyclophosphamide, doxorubicin, vincristine, and prednisone and cures approximately $60 \%$ of the patients [10]. The key compounds in R-CHOP are the topoisomerase II inhibitor doxorubicin and the monoclonal antibody rituximab. Doxorubicin is an anthracycline that induces DNA damage, and its addition to the regimen increased the 10year overall survival of $20 \%[11,12]$. Rituximab targets the B-cell surface protein CD20, and both activate immune response and induce apoptosis via p38 MAP-kinase signaling pathway $[13,14]$. Addition of rituximab to $\mathrm{CHOP}$ increased the 5-year overall survival by $10 \%$ [15-19]. Even though several genes, such as TP53, STAT3/6, CDKN2A, and $E Z H 2$, have been suggested to confer resistance to RCHOP $[20,21]$, there is no clinically effective treatment available for resistant patients. Given that DLBCL is the most common aggressive lymphoid cancer [22] and that patients who are not cured with $\mathrm{R}-\mathrm{CHOP}$ have dismal prognosis, novel strategies to overcome $\mathrm{R}-\mathrm{CHOP}$ resistance are urgently needed.

Our observations from screening 60 epigenetic inhibitors in four DLBCL cell lines revealed HDAC and HMT inhibitors as particularly effective in sensitizing these cell lines to doxorubicin and rituximab. Our results further show that epigenetic sensitization is achieved at lower doses than epigenetic cytotoxicity, potentially causing less severe side effects in clinical settings. Thus, the herein identified inhibitors are clinically promising candidates for combination treatment of resistant of refractory DLBCL patients.

To further describe mechanisms involved in the effectiveness of epigenetic compounds, we generated transcriptome sequencing and immunofluorescence data from DLBCL cell lines before and after treatment. Analyses of these data highlighted dysregulation of DNA repair as a key mechanism for episensitization. To facilitate exploiting our data and results, we have implemented an interactive Results Explorer tool (http://app. anduril.org/DLBCL_DSRT).

\section{Results}

High-throughput multi-step drug combination screening To systematically investigate the reprogramming ability of multiple epigenetic compounds, we have designed a high-throughput screening protocol that uses automated liquid handling to pretreat suspension cells with epigenetic inhibitors before exposing them to doxorubicin and rituximab, as representatives of standard treatment. The protocol comprises three main steps. First, cells are seeded on two replicate sets of microplates with previously administered reprogramming compounds. A 10, 000 -fold concentration range is used to test each epigenetic inhibitor, to determine the optimal dose inducing sensitization. Second, cells are incubated with the compounds for either 1 or 3 days (pilot experiment, Additional file 1: Figure S1), or for 9 days using on-plate passaging protocol (Fig. 1). This allows estimation of the time needed by each compound to induce cellular reprogramming.

The 9-day pretreatment is too long for cells to survive without fresh media. Thus, we developed a robotic-based protocol for on-plate cell passaging. After pretreatment, one plate set is treated with rituximab and doxorubicin while keeping another pretreated plate set as control. Third, we measured cell viability and estimated the sensitization induced by each compound using a reprogramming score, i.e., the maximum difference in cell viability between the effect of the epigenetic inhibitor alone and the effect of the epigenetic inhibitor followed by administration of rituximab and doxorubicin.

\section{Epigenetic inhibitors sensitize DLBCL cell lines to doxorubicin and rituximab}

We applied the screening protocol to investigate whether epigenetic inhibitors are able to sensitize four DLBCL cell lines (Oci-Ly-3, Riva-I, Su-Dhl-4, and OciLy-19) to doxorubicin and rituximab. We first conducted a pilot screening using short pretreatment times up to 3 days (described in the Additional file 1: Figure S3). This pilot experiment indicated that increasing the length of the pretreatment window enhances the reprogramming ability of epigenetic inhibitors. Thus, we increased the pretreatment time to 9 days in the main drug screening assay.

With the 9-day reprogramming, HDAC inhibitors sensitized all cell lines (Fig. 2c), while BRD and HMT inhibitors induced sensitization in three cell lines. Oci-Ly-3 cells were the most responsive, with 20 out of the 60 epigenetic inhibitors able to sensitize to doxorubicin and rituximab. Oci-Ly-19 and Su-Dhl-4 cells were sensitized by nine and 10 inhibitors, respectively. Riva-I was the most resistant cell line and was reprogrammed only by three inhibitors. Varying response to the inhibitors was expected due to heterogenous nature of DLBCL and because compounds such as HDAC inhibitors are known to have different efficacy depending on cancer type and dosage [23]. The optimal concentration at which each compound induced reprogramming was always lower 


Day $\mathbf{0}$
Cell seeding on
precoated plates

than the concentration at which the same compound would induce cytotoxicity. Dose response curves are available in the Results Explorer.

\section{Epigenetic reprogramming acts synergistically with rituximab and doxorubicin}

We conducted a drug synergy assay to validate the observed reprogramming effect of the 10 most potent inhibitors. Compounds with three or more hits, i.e., belinostat, entinostat, and I-BET151, were tested for synergy in all four cell lines, whereas the other compounds were administered only to those cell lines they reprogrammed in the screening. This validation assay followed the design shown in Fig. 1, but varying concentrations of the epigenetic inhibitors, as well as of rituximab and doxorubicin, were now used (see Additional file 1 and Additional file 2: Table S1).

The synergy plots are available through the Results Explorer, while Fig. 3 summarizes the synergy scores. A score close to zero indicates that the killing effect of an inhibitor is independent from the killing effect of doxorubicin and rituximab, whereas a high score indicates a synergistic effect [24]. None of the compounds showed high negative scores, which indicates lack of antagonistic effects. The highest synergy scores were observed in compounds targeting HDACs (vorinostat, entinostat, resminostat, belinostat) or HMTs (pinometostat, tazemetostat, SGC0946). The most potent sensitization effects were induced by the entinostat and tazemetostat. Inhibition of BRDs showed lower synergy.

This validation experiment confirmed the findings in the original screen: Oci-Ly-3 cells were the most responsive to reprogramming, and HDAC and HMT inhibitors sensitized them to rituximab and doxorubicin. Su-Dhl-4 and Oci-Ly-19 cells responded to more than one synergistic inhibitor, whereas belinostat was the only compound synergistically reprogramming Riva-I cells.

\section{Epigenetic sensitization to doxorubicin is achieved through reprogramming of DNA repair mechanisms} Enhanced DNA repair is one of the key resistance mechanisms for doxorubicin [25], so we hypothesized that the observed sensitization might be due to impaired repair. Immunofluorescence imaging of cCasp3, gH2Ax, and RAD51 is a well-established and quantitative approach for assaying DNA repair: cCasp3 is an early and specific indicator of apoptosis, gH2Ax measures the amount of DNA damage, and RAD51 indicates that homologous recombination is actively repairing DNA. Hence, we used immunofluorescence imaging of these three proteins (Fig. 4a) to investigate DNA repair pathways affected by entinostat, tazemetostat, belinostat, and vorinostat (which showed high synergy with doxorubicin and rituximab). Of note, these compounds are also clinically relevant as belinostat and vorinostat received FDA-approval for the treatment of patients with relapsed or refractory peripheral T-cell lymphoma and cutaneous T-cell lymphoma, while entinostat and tazemetostat have obtained FDA "Breakthrough" and "Fast Track" designations, respectively.

Cells treated with the HDAC inhibitors (entinostat, belinostat, vorinostat) showed reduced RAD51 foci formation (Additional file 1: Figure S4), suggesting impaired homologous recombination (HR). Nonhomologous end joining (NHEJ) was upregulated in cells treated with HDAC inhibitors, which was expected as NHEJ is often seen as a compensatory effect for 


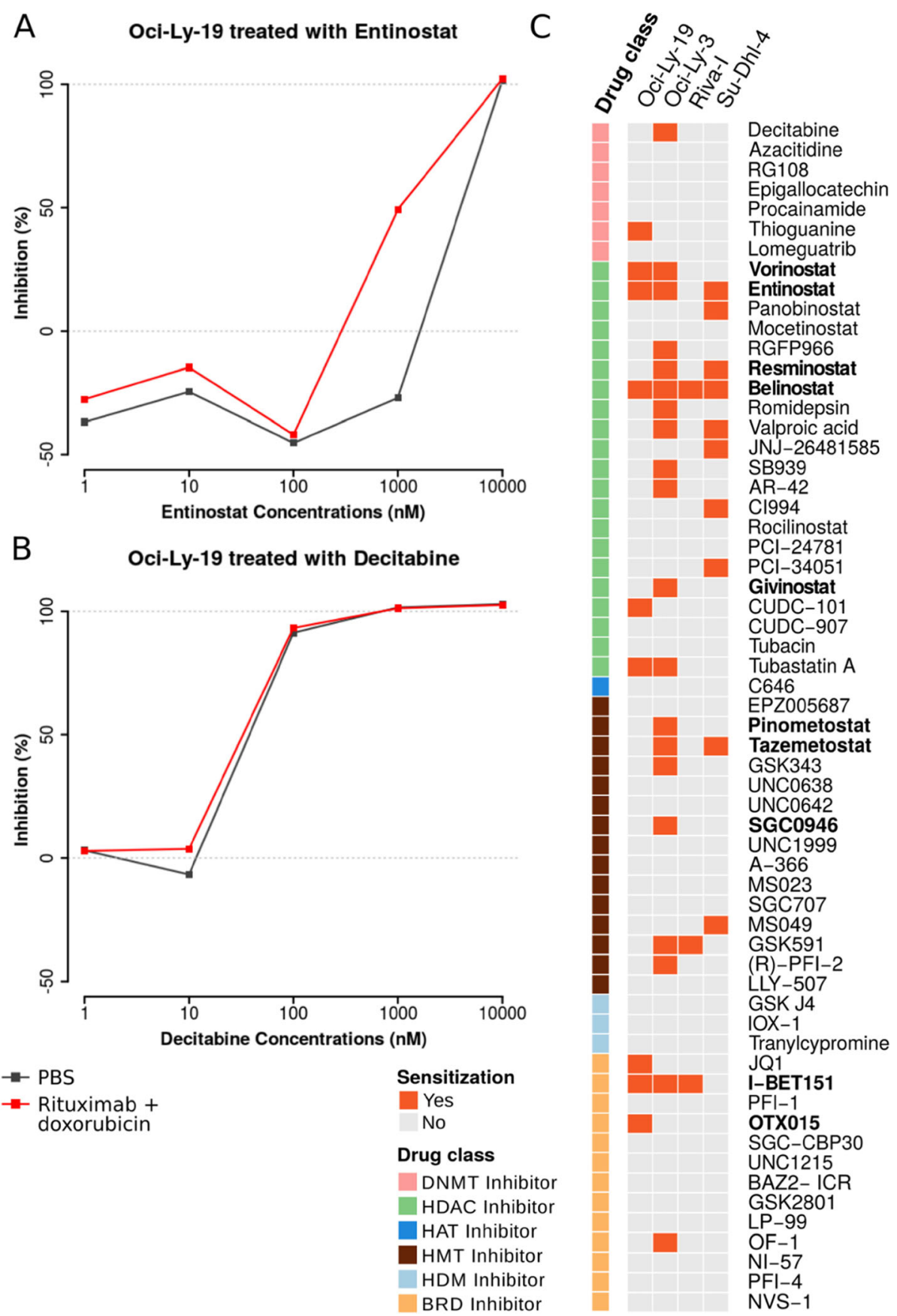

Fig. 2 Results of the high-throughput multi-step drug combination screening with 9 days of epigenetic pretreatment. a An example of a compound that induced sensitization to rituximab and doxorubicin vs. phosphate-buffered saline (PBS). The combination induced $50 \%$ growth inhibition at $1000 \mathrm{nM}$ concentration, which is not seen with monotreatment with entinostat. The main aim of this report is to identify such sensitizising compounds. $\mathbf{b}$ An example of a compound that does not sensitize cells to rituximab and doxorubicin, but has a cytotoxic effect. $\mathbf{c}$ Summary of the reprogramming screening hits of 60 compounds across four DLBCL cell lines. Reprogramming scores above a threshold of $30 \%$ (see Materials and Methods) and whose dose-response curve passed quality inspection are considered as hits and marked in orange. Ten compounds, marked in bold, were selected for the synergy assay based on their reprogramming potential and mechanisms of action. All dose response curves are available in Results Explorer (http://app.anduril.org/DLBCL_DSRT)

impaired HR. Cells treated with the HMT inhibitor tazemetostat did not show significant effect on DNA repair pathways.
These results support the hypothesis that HDAC inhibitor sensitization occurs by impairing HR repair, as shown in Fig. 4b, c. Entinostat alone does not 


\begin{tabular}{|c|c|c|c|c|c|c|c|c|c|c|c|}
\hline & Vorinostat & Entinostat & Resminost. & Belinostat & Givinostat & Pinometost. & Tazemetost. & SGC0946 & I-BET151 & OTX015 & \multirow{2}{*}{$\begin{array}{l}\text { Synergy } \\
\text { score } \\
20\end{array}$} \\
\hline Oci-Ly-19 & 4.97 & 6.80 & & 0.58 & & & & & 2.53 & 1.33 & \\
\hline Oci-Ly-3 & & 2.83 & 4.80 & 0.71 & 1.30 & 6.98 & 2.40 & 7.43 & 0.76 & & \\
\hline Riva-I & & -0.09 & & 5.17 & & & & & -0.28 & & 10 \\
\hline Su-Dhl-4 & & 21.32 & & -0.18 & & & 14.22 & & 3.83 & & 0 \\
\hline & 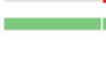 & $H D A$ & $A C$ inhibitc & tors & 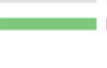 & & MT inhibito & & BRD in & hibitors & -10 \\
\hline
\end{tabular}

affect the number of cells positive for double strand breaks, apoptosis, or HR, compared to the untreated control. However, the response to doxorubicin was strikingly different in cells treated with entinostat compared to untreated cells. The control cells were able to repair DNA damage due to high HR activity (Fig. 4c, blue bar) and thus avoid apoptosis.
Transcriptomic analysis identifies disruption of DNA repair, cell cycle, and apoptosis as potential mechanisms behind epigenetic sensitization

To further characterize the molecular mechanisms affected by the observed epigenetic sensitization, we performed RNA-seq of the four cell lines before and after treating them with belinostat, entinostat, vorinostat, and
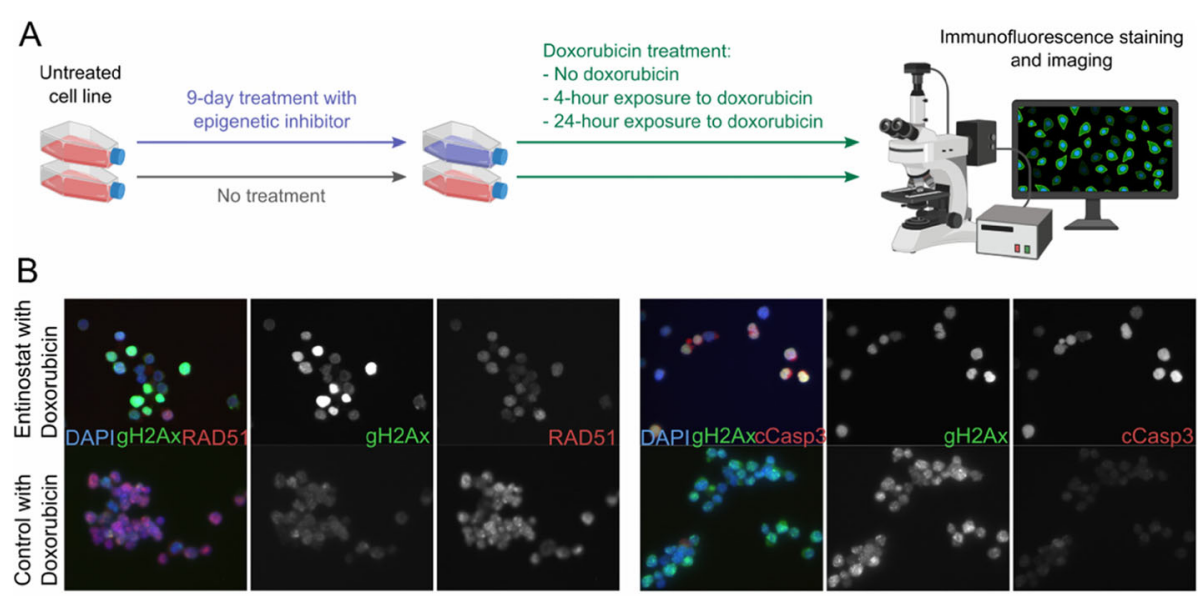

C

No Doxorubicin - No induced DNA damage

Doxorubicin (4h) - Induced DNA damage
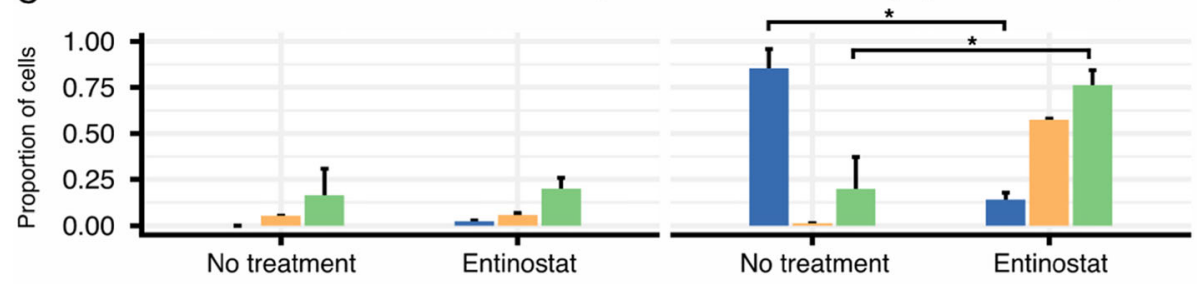

RAD51 cCasp3 $\square \mathrm{gH} 2 \mathrm{Ax}$

Fig. 4 Effect of doxorubicin treatment in Oci-Ly-19 after entinostat treatment on DNA repair mechanisms quantified by immunofluorescence assay. a Schematic representation of the experimental procedure prior immunofluorescence staining. Cell lines were treated with epigenetic inhibitors (i.e., entinostat, belinostat, vorinostat, or tazemetostat) at days 0, 3, 6, and 9, while a copy was kept untreated as control. After 9 days, both treated and control cell lines either received no doxorubicin or were exposed to doxorubicin for 4 and $24 \mathrm{~h}$. Cells were then stained using immunofluorescent antibodies and imaged. Cells not receiving any treatment served as negative control, while cells not pretreated with epigenetic inhibitors but treated with doxorubicin served as positive control. b Immunofluorescence images of doxorubicin treated cells after entinostat treatment (above) and untreated (below). Composite image includes also DAPI (blue). c Quantification of proportion of cells positive for each marker in each image. The markers shown quantify apoptosis (cCasp3), DNA damage (gH2Ax), and homologous recombination (RAD51) (see Methods). Entinostat-induced sensitization to exogenous DNA damage is detected as reduced RAD51 foci, increased cCasp3 positivity and increased gamma-H2AX positivity in doxorubicin-treated cells. Asterisks indicate significant regulation $(p<0.05)$. All measurements from the immunofluorescence assay are shown in Additional file 1: Figure S4 
tazemetostat (Additional file 1: Figure S5A). Differentially expressed genes (DEGs) between treated and untreated cells are shown in Additional file 1: Figure S5 B-E, and can be browsed in the Results Explorer. Gene expression landscape across cell lines and treatment conditions is shown in Additional file 1: Figure S6.

We used DEGs from each successfully reprogrammed combination and performed pathway enrichment analysis to explore the reprogramming mechanisms. An overview of the top pathways identified using WikiPathways database is shown in Fig. 5. All pathway results including $p$ values and pathway-specific DEGs for KEGG, Reactome, and WikiPathways are provided in Additional file 3: Table S2. All sensitized combinations showed changes in immune response mechanisms. This was expected since DLBCL originates from B-cells, which produce antibodies in the adaptive immune system [26]. Our analysis further revealed the major histocompatibility complex (Additional file 3: Table S2) as one of the pathways most affected by HDAC inhibitors, which is in line with a study by Eckschlager and colleagues [23].

DNA damage and repair mechanisms were dysregulated in Su-Dhl-4 and Oci-Ly-19 cells treated with entinostat, as well as in Su-Dhl-4 cells treated with tazemetostat. When comparing untreated and treated conditions for all successfully sensitized cell line and inhibitor combinations, we identified DEGs belonging to HR, NHEJ, and other DNA repair pathways (Additional file 4: Table S3). While DEGs involved in NHEJ were identified only in Su-Dhl-4 cells treated with entinostat, HR genes were differentially expressed both in Su-Dhl-4 cells treated with entinostat and tazemetostat, as well as in Oci-Ly-19 cells treated with entinostat and vorinostat. In particular, these combinations showed downregulation of XRCC2 and $P O L Q$. $X R C C 2$ is essential for the proper functioning of $\mathrm{HR}$ [27], and knockdown of $P O L Q$ in HR-deficient tumors enhances cell death [28]. Thus, decreased expression of both genes may contribute to the sensitization of doxorubicin-resistant DLBCLs. Additionally, Su-Dhl-4 cells treated with entinostat or tazemetostat also showed downregulation of RAD51, RAD54L, BRCA2, and three Fanconi anemia genes (FANCA, FANCB, and $F A N C M$ ). BRCA1 expression was suppressed in $\mathrm{Su}-\mathrm{Dhl}-4$ cells in response to entinostat. In Riva-I cells, belinostat-induced differential expression did not have an impact on the expression of any HR gene, making it the only cell line reprogrammed by inhibition of HDACs and not showing transcriptional changes in DNA repair.

Disruption of cell cycle and DNA replication were also mechanisms identified in several sensitized combinations

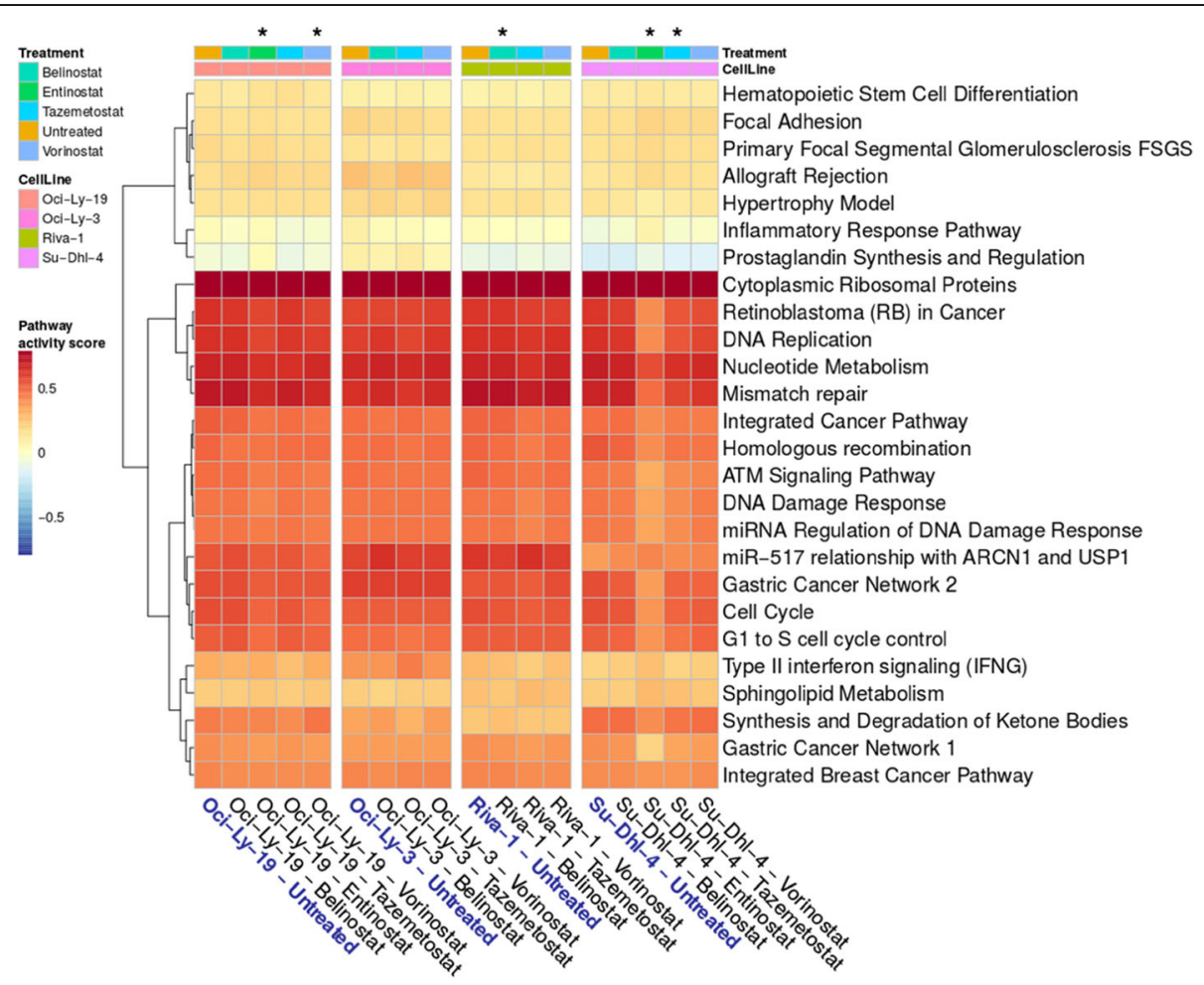

Fig. 5 Summary of pathway analysis results for all cell lines and conditions. Columns represent the cell line-treatment combination (untreated conditions are marked in blue). Sensitizing combinations are marked by an asterisk. The rows include the top pathways identified from WikiPathways database. All pathway analysis results are available in Additional file 3: Table S2 
(i.e., Su-Dhl-4 and Oci-Ly-19 cells treated with entinostat, and Su-Dhl-4 cells treated with tazemetostat). In particular, treatment of Su-Dhl-4 cells with entinostat led to upregulation of CDKN1A, an HDAC inhibitor mechanism previously suggested to induce cell cycle arrest [23].

Other pathways identified in our analysis included cell adhesion (altered in all sensitized combinations except Riva-I cells treated with belinostat) and TGF- $\beta$ signaling (disrupted in Oci-Ly-19 cells treated with either entinostat or vorinostat, and in Su-Dhl-4 cells treated with entinostat). Death receptors and ligands belonging to the TNF and TNF-receptor superfamilies were differentially expressed in all sensitized combinations. Indeed, HDAC inhibitors have been demonstrated to affect apoptosis through dysregulation of such protein families [23]. Pathway analysis also showed disruption of the apoptotic pathway in Riva-I cells treated with belinostat (Additional file 3: Table S2).

\section{Whole exome-sequencing suggests genomic variants relevant to drug response}

To analyze the genomic variants of the cell lines, we performed whole exome sequencing. We decided to produce sequencing data directly from our cells to avoid bias due to genetic evolution of the cell lines [29]. This analysis identified somatic mutations and known germline single nucleotide polymorphisms (SNPs) that could influence drug response or the synergistic effect of epigenetic sensitizing compounds used in combination with doxorubicin and rituximab (Additional file 5: Table S4).

Among epigenetic genes, EZH2 was found mutated (p.Y590S, missense mutation) in Su-Dhl-4, the cell line responding to the EZH2 inhibitor tazemetostat. Interestingly, this mutation has been reported to increase sensitivity to EZH2 inhibitors [30]. We also observed a truncating mutation (p.R1322X) in CREBBP, impairing histone acetylation, and transcriptional regulation of its targets [31] was shared in Riva-I and Oci-Ly-19 (subclonal). Truncation of CREBBP is acquired in relapsed acute lymphocytic leukemia [31], further supporting its important role in mediating chemotherapy resistance in lymphoid malignancies. In the same gene category, we observed a truncating mutation in the ARID1A gene (p.Q474X) of Riva-I cells. ARID1A was previously reported to encode for a critical transcription factor in the absence of HDAC6 [32]. It is also the most commonly mutated and functionally disrupted component of the tumor suppressor chromatin remodeling SWI/SNF complex and thereby has been reported as an important epigenetic modulator [33] and contributor to genetic and genomic instability and response to DNA damaging agents [34].

All our cell lines harbor at least one potentially functional mutation in genes encoding epigenetic enzymes targeted in this study or in genes potentially contributing to response to epigenetic inhibitory drugs reported in other studies. Notably, the majority of these genes are among the most recurrently altered drivers in DLBCL (MYD88 in 18\%, CREBBP, ARID1A, and TP53 in $10 \%$, and $E Z H 2$ in $6 \%$ of patients) [35]. A comprehensive genomic profiling of the cell lines is reported in the Additional file 1.

\section{Increased CD20 expression does not sensitize all cell lines to rituximab}

Next, we investigated whether rituximab sensitization in response to epigenetic modifiers resulted from increased expression of the MS4A1 gene encoding for CD20. All four untreated cell lines express MS4A1 (with Oci-Ly-19 showing the lowest expression), but none of the four tested epigenetic compounds was able to upregulate MS4A1 expression. However, CD40, a key effector of CD20 on B cells [36], was inhibited in Oci-Ly-19 cells in response to vorinostat or tazemetostat.

We also examined if sensitization to rituximab could be due to increased expression of CD20 on the cell surface. Two compounds shown to support CD20 transport to the cell membrane were tested using our highthroughput screening (Additional file 1: Figure S7). Rifampicin, an antibiotic shown to restore efficacy of antiCD20 antibodies [37], was successful only on Oci-Ly-19 cells. Suramin, a small molecule that inhibits CD40, sensitized the rest of the cell lines. However, since this sensitization was observed at different pretreatment times for different cell lines, we cannot conclude that upregulation of $\mathrm{CD} 20$ expression is required for sensitization to rituximab.

When comparing the expression of rituximab-related genes [38] across untreated cell lines, we observed that Oci-Ly-3 presented a unique transcriptomic profile. Specifically, Oci-Ly-3 was the only cell line showing overexpression of CXCL13, a B cell-attracting chemokine [39], and downregulation of $C D 27$, a proapoptotic gene previously shown to link HDACs activation and cell cycle arrest [40].

\section{Discussion}

Finding effective treatment options for relapsed and refractory malignancies is a major challenge in cancer therapy. Due to the plasticity of the epigenome, epigenetic inhibitors are a particularly interesting class of compounds to sensitize cancer cells to standard therapeutic options [41]. Hence, we designed a high-throughput experimental protocol that allows identifying epigenetic inhibitors able to (re)sensitize cancer cells to standard therapeutic agents. This approach is a major step towards finding clinically useful and effective drug combinations. Moreover, the customizable plate layout and the 
ability to vary experiment duration by on-plate cell passaging make this protocol suitable for testing multi-step delivery for other drug combinations.

As a case study, we tested our protocol on DLBCL cell lines that represent the heterogeneity of the disease. We demonstrated how this experimental approach helped to systematically evaluate the sensitization power of 60 epigenetic inhibitors. Our results indicate that most of these inhibitors require several days to effectively induce reprogramming. Only a few compounds, mainly HDAC inhibitors, were able to sensitize cell lines within 1 and 3 days of pretreatment. Increasing the pretreatment length allowed us to identify additional actionable mechanisms that would be missed with a shorter assay. For instance, DNMT inhibitors are expected to be slow acting, since passive demethylation requires several cell cycles. Pairing 9 days of pretreatment time with multiple doses of epigenetic inhibitors sensitized all cell lines to doxorubicin and rituximab, the key compounds of R-CHOP. This suggests that epigenetic reprogramming is an effective option across all molecular subtypes in DLBCL.

Importantly, this study demonstrates that epigenetic drugs induced sensitization at much lower doses than those required for cytotoxicity, suggesting that when epigenetic drugs are used to sensitize rather than kill cancer cells, they are likely to cause less severe sideeffects. Based on the tested pretreatment times, we propose that epigenetic inhibitors should be administered in a reprogramming mode, i.e., several doses and days before chemotherapy, rather than simultaneously with chemotherapy.

In this study, HDAC inhibitors belinostat, entinostat, vorinostat, and resminostat, as well as HMT inhibitors tazemetostat, pinometostat, and SGC0946, were the most potent epigenetic drugs to sensitize cancer cells to doxorubicin and rituximab. Both classes of inhibitors are reported to be well-tolerated in clinical trials [23, 42, 43]. Further pre-clinical investigation of their reprogramming potential to identify the correct time and dose for epigenetic sensitization will broaden the use of these inhibitors as pretreatment options on chemo-resistant cancers.

This protocol allowed to identify the right dose and pretreatment length for multiple epigenetic inhibitors. We then used these parameters to investigate the mechanisms behind epigenetic reprogramming through transcriptome analysis. Each cell line achieved reprogramming through a different combination of altered pathways, but we identified dysregulation of DNA repair (especially HR), disruption of cell cycle, and effects on apoptosis as the main drivers of sensitization. This adds on previous observations on the mode of action of HDAC and HMT inhibitors [23, 44]. Resistance to DNA damaging agents, such as doxorubicin [45], has often been associated with upregulation of HR-mediated DNA repair.
Our results show that belinostat, entinostat, vorinostat, and tazemetostat can sensitize DLBCL cells via disruption of the HR pathway. Further, sensitization by these inhibitors affected the expression of several DNA repair genes, such as $X R C C 2$ and $P O L Q$, both downregulated in most sensitized combinations. These findings argue that epigenetic inhibitors could also revert resistance to a wider class of DNA damaging agents, such as platinum-based regimens and radiotherapy.

Belinostat, as the broadest pan-HDAC inhibitor in our collection, sensitized cells with very low evidence of $\mathrm{HR}$ dysregulation but strong truncation of ARID1A transcription factor, critical for the cell's survival in the absence of HDAC6 [32]. In depth analysis of pan-HDAC effects on a different cell line cohort might highlight alternative mechanisms of action, such as disruption of apoptosis observed in belinostat-treated Riva-I.

Even though we found a clear link between epigenetic modifiers and sensitization to doxorubicin, we were unable to find the same for rituximab. The major reasons are the undefined mechanisms of action of CD20 and the absence of known regulatory pathways modulating this protein.

Through the genomic characterization of our cell lines, we were able to highlight commonly mutated genes among DLBCL patients [35] with functional and clinical relevance. This opens a line of pre-clinical biomarker investigation for future personalized sensitization therapy. The most striking mutation was identified on gene $E Z H 2$ in the GCB cell line Su-Dhl-4, the best responder to EZH2-inhibitor tazemetostat. This EZH2 inhibitor has the highest efficacy in EZH2-mutated DLBCL patients belonging to the GCB subtype [46], and the mutation found in Su-Dhl-4 cells has been reported as a potential biomarker for response to EZH2 inhibitors [47]. Moreover, we observed high tazemetostat-driven dysregulation of cell cycle genes in these cells, potentially causing cell cycle arrest, a mechanism suggested by Knutson et al. [44]. Altogether, these results suggest that R-CHOP resistant DLBCL patients with EZH2 mutations could benefit from tazemetostat pretreatment followed by R-CHOP re-challenge.

Our study has some limitations. First, when we created the compound library, only few HDM and HAT inhibitors were available. In our experiments, these compounds were not able to induce reprogramming, but testing a more extensive inhibitor collection might identify reprogramming mechanisms targeting HDM and HAT enzymes. Second, even though all DLBCL subtypes were represented in our study, the number of cell lines included was just enough to show the value of the proposed protocol but relatively small to propose clinical strategies or biomarkers with high confidence. Future studies can however use this protocol and increase the 
number of samples and drugs. Third, since our readout was cytotoxicity and not tumor immunity, the impact of tumor microenvironment could not be tested in this model; hence, the observed epigenetic reprogramming of rituximab resistance may be only partially detected. Fourth, some HDAC inhibitors have been shown to have off-target effects [48], which cannot be separated from the on-target effects in the transcriptomic analyses due to the experimental design we have employed. Such pleiotropic effects, however, do not impact our combination therapy results herein but may pose challenges for tolerability of the combination treatment and identification of specific biomarkers in future studies. Last, even though our results suggest that epigenetic inhibitors can be useful in a clinical setting, a more detailed analysis is needed to estimate optimal dose and treatment duration in vivo.

\section{Conclusions}

Taken together, in this contribution we report a novel methodology to screen sensitization effect of nonsimultaneous drug combinations. It is also among the few studies investigating the use of epigenetic drugs as sensitizing agents, instead of using them as mono- or combination therapy, and it is the first to do so in a systematic and high-throughput manner. The application of this method to overcome $\mathrm{R}-\mathrm{CHOP}$ resistance further supports the use of epigenetic reprogramming in sensitizing DLBCL to standard immunochemotherapy combinations.

\section{Materials and methods}

An overview of the main materials and methods is reported below. For a detailed description of experimental protocols and bioinformatic pipelines, see the Additional file 1.

\section{Compound collection}

We curated the compound library by manually searching literature and providers for compounds inhibiting epigenetic enzymes. We collected both FDA-approved compounds and probes targeting DNMT $(n=7)$, $\operatorname{HDAC}(n=21), \operatorname{HAT}(n=1), \operatorname{HMT}(n=15), \operatorname{HDM}(n$ $=3)$, and BRD $(n=13)$. The full list of compounds used in each experiment is available in Fig. 2 and Additional file 1: Figure S3, while concentration ranges are listed in Additional file 2: Table S1.

\section{Cell lines}

We performed our epigenetic screening on four DLBCL cell lines, representative of all DLBCL subtypes [49] and with varying response to rituximab and doxorubicin (Additional file 1: Figure S2). Su-Dhl-4 belongs to the GCB subtype [50], Oci-Ly-3 and Riva-I to the ABC subtype [50], while Oci-Ly-19 is unclassified.

\section{Screening procedure and parameters}

Compounds were dissolved in DMSO and added to the assay plates using a Labcyte Echo 550 acoustic dispenser. The highest dose concentration was as advised by the supplier followed by four tenfold dilutions.

Plate layout design included randomized positive (benzethonium chloride, $\mathrm{BzCl}$, Sigma-Aldrich) and negative (DMSO, Sigma-Aldrich) controls. Compound plates were stored under inert nitrogen gas in StoragePods (Roylan Developments) until needed. Cells were seeded using BioTek MultiFlo FX Random Access Dispenser, at 3000 cells/well (Riva-I, Su-Dhl-4, Oci-Ly-19) or 4000 cells/well (Oci-Ly-3) in $25 \mu \mathrm{L}$ ( 1 and 3 days of pretreatment time) or $40 \mu \mathrm{L}$ (9 days of pretreatment time). Cell plates were incubated in a Thermo Scientific Cytomat $10 \mathrm{C}$ incubator at $37^{\circ} \mathrm{C}$ and $5 \%$ $\mathrm{CO}_{2}$. Plates undergoing 9 days of pretreatment had a Labcyte microclime lid to reduce media evaporation during the incubation period. During the longest pretreatment time, cells were passaged every third day (i.e. on days 3,6 , and 9) directly in-plate with pretreatment drugs added to the new media. Since all cell lines grew in suspension, plates were spun down before passaging. In-plate passaging was then performed with a Beckman Coulter Biomek FXp pipetting device fitted with a 384 multichannel head. The BioMek FXp protocol included the following steps:

1. Aspirate $20 \mu \mathrm{L}$ of old media from the culture plate (without touching the cells collected at the bottom of the well) and discard it.

2. Aspirate $20 \mu \mathrm{L}$ of fresh media from the plate with drugs in media and dispense it on the cells.

3. Resuspend the cells by mixing with $20 \mu \mathrm{L}$ volume five times.

4. Aspirate $20 \mu \mathrm{L}$ of old media and cells from the culture plate and discard it.

5. Aspirate $20 \mu \mathrm{L}$ of fresh media from the plate with drugs in media and dispense it on the cells.

With this procedure, roughly $3 / 4$ of the media was exchanged while removing half of the cells from each well.

After pretreatment, half of the plates were treated with a fixed dose of rituximab (MabThera, diluted in phosphatebuffered saline (PBS). Roche) and doxorubicin (diluted in PBS. Sigma-Aldrich), while the other half received only PBS as control. The concentrations of rituximab and doxorubicin were determined through a drug combination assay and are listed in Additional file 2: Table S1. After treatment, cells were incubated for $48 \mathrm{~h}$. Finally, cell viability was measured with Promega CellTiter-Glo reagent and BMG LABTECH FLUOstar Omega plate reader.

\section{Analysis of DNA damage, DNA repair, and apoptosis}

We used an immunofluorescence assay to measure doxorubicin-induced DNA damage as double-strand 
breaks (detected as $\gamma \mathrm{H} 2 \mathrm{Ax}$ foci), efficiency of DNA repair via homologous recombination (detected as RAD51 foci) and non-homologous end joining (detected 53BP1 foci), and apoptosis (detected as cleaved-Casp3). Measurements obtained from untreated cells were compared with those from cells pretreated with belinostat, entinostat, vorinostat, or tazemetostat.

\section{Whole exome and RNA sequencing}

All untreated cell lines were analyzed by whole exome sequencing to identify mutations linked to epigenetic sensitization. We also performed RNA sequencing for the four cell lines untreated, as well as treated with belinostat, entinostat, vorinostat, or tazemetostat, to identify transcriptomic changes induced by epigenetic reprogramming (see Additional files 1 and 6).

\section{Supplementary information}

Supplementary information accompanies this paper at https://doi.org/10. 1186/s13148-019-0781-3.

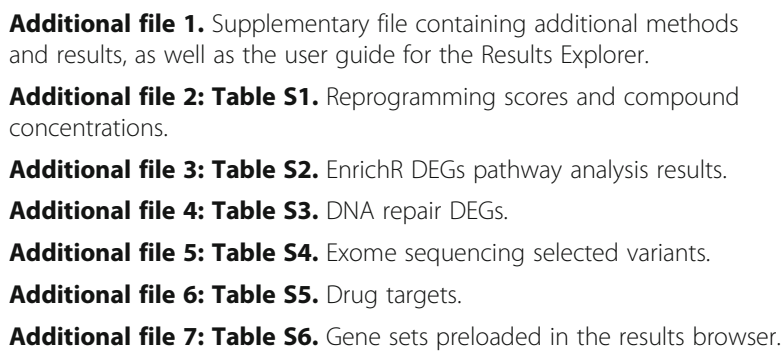

Additional file 1. Supplementary file containing additional methods and results, as well as the user guide for the Results Explorer.

Additional file 2: Table S1. Reprogramming scores and compound concentrations.

Additional file 3: Table S2. EnrichR DEGs pathway analysis results.

Additional file 4: Table S3. DNA repair DEGs.

Additional file 5: Table S4. Exome sequencing selected variants.

Additional file 6: Table S5. Drug targets.

Additional file 7: Table S6. Gene sets preloaded in the results browser.

\section{Abbreviations}

ABC: Activated B-cell; BRD: Bromodomain; DEG: Differentially expressed gene; DLBCL: Diffuse large B-cell lymphoma; DNMT: DNA methyltransferases; GCB: Germinal center; HAT: Histone acetyltransferase; HDAC: Histone deacetylase; HDM: Histone demethylase; HMT: Histone methyltransferase; HR: Homologous recombination; NHEJ: Non-homologous end joining; RCHOP: Standard therapy for DLBCL including rituximab, cyclophosphamide, doxorubicin, vincristine, and prednisone; SNP: Single-nucleotide polymorphism

\section{Acknowledgements}

The authors thank Dr. Karen Dybkær, for providing us the cell lines used in this study, Structural Genomics Consortium (SGC) for sharing the 16 compounds tested in the 9-day pretreatment, and IT Center for Science (CSC) for computing resources. The authors also thank Anne Aarnio, Marika Tuukkanen, and Kirsi Jäntti for their guidance and technical assistance, and Jani Saarela for the helpful discussions about drug screening data analysis.

\section{Authors' contributions}

C.F. and J.C. contributed equally to the study and wrote the manuscript. C.F. and J.C. conceptualized the study together with S.H., R.L., S.L., K.W., S.-K.L., and L.T; C.F., J.C., L.T., V.R., and M.T. performed experiments; R.L., S-K.L., S.L., and S.H. supervised the study; L.K., S.L., and S.H. acquired funding. All authors read and edited the paper.

\section{Funding}

C.F. and J.C. have been supported by the Doctoral Program in Biomedicine This project has received funding from the Academy of Finland (to L.K. S.H., and S.L.), the Sigrid Jusélius Foundation (S.H. and S.L.), Finnish Cancer Association (S.H. and S.L.), and Helsinki University Hospital (S.L.).

\section{Availability of data and materials}

All raw fasta files from RNA-Seq and WES are available at SRA [51] (accession number PRJNA517451). All our processed results can be browsed through our Results Explorer tool (http://app.anduril.org/DLBCL_DSRT).

Ethics approval and consent to participate

Not applicable.

\section{Consent for publication}

Not applicable.

\section{Competing interests}

The authors declare that they have no competing interests.

\section{Author details}

${ }^{1}$ Research Program in Systems Oncology, Faculty of Medicine, University of Helsinki, PO Box 63, Helsinki, Finland. ${ }^{2}$ Institute for Molecular Medicine Finland (FIMM), University of Helsinki, Helsinki, Finland. ${ }^{3}$ Department of Oncology, Helsinki University Hospital Cancer Center, Helsinki, Finland. ${ }^{4}$ Research Program in Applied Tumor Genetics, Faculty of Medicine, University of Helsinki, Helsinki, Finland.

Received: 1 August 2019 Accepted: 17 November 2019

Published online: 11 December 2019

\section{References}

1. Barrero MJ, Boué S, Izpisúa Belmonte JC. Epigenetic Mechanisms that regulate cell identity. Cell Stem Cell. 2010;7:565-70.

2. Zoghbi HY, Beaudet AL. Epigenetics and human disease. Cold Spring Harb Perspect Biol. 2016;

3. Biswas S, Rao CM. Epigenetics in cancer: fundamentals and beyond. Pharmacol Ther. 2017;173:118-34.

4. Flavahan WA, Gaskell E, Bernstein BE. Epigenetic plasticity and the hallmarks of cancer. Science (80- ). 2017;

5. Housman G, Byler S, Heerboth S, Lapinska K, Longacre M, Snyder N, et al. Drug resistance in cancer: an overview. Cancers (Basel). 2014;6:1769-92.

6. Ahuja N, Sharma AR, Baylin SB. Epigenetic therapeutics: a new weapon in the war against cancer. Annu Rev Med. 2016;67:73-89.

7. Clozel T, Yang S, Elstrom RL, Tam W, Martin P, Kormaksson M, et al. Mechanism-based epigenetic chemosensitization therapy of diffuse large Bcell lymphoma. Cancer Discov. 2013;3:1002-19.

8. Sharma SV, Lee DY, Li B, Quinlan MP, Takahashi F, Maheswaran S, et al. A chromatin-mediated reversible drug-tolerant state in cancer cell subpopulations. Cell. 2010;141:69-80.

9. Kurimoto M, Matsuoka H, Hanaoka N, Uneda S, Murayama T, Sonoki T, et al. Pretreatment of leukemic cells with low-dose decitabine markedly enhances the cytotoxicity of gemtuzumab ozogamicin. Leukemia. 2013;27:233-5.

10. Roschewski M, Staudt LM, Wilson WH. Diffuse large B-cell lymphoma treatment approaches in the molecular era. Nat Rev Clin Oncol. 2014;11:12-23.

11. Goy A. Succeeding in breaking the R-CHOP ceiling in DLBCL: learning from negative trials. J Clin Oncol [Internet]. 2017;35:3519-22. Available from. https://doi.org/10.1200/JCO.2017.74.7360.

12. Lee KW, Kim DY, Yun T, Kim DW, Kim TY, Yoon SS, et al. Doxorubicin-based chemotherapy for diffuse large B-cell lymphoma in elderly patients: comparison of treatment outcomes between young and elderly patients and the significance of doxorubicin dosage. Cancer. 2003;98:2651-6.

13. Singh V, Gupta D, Arora R, Tripathi RP, Almasan A, Macklis RM. Surface levels of CD20 determine anti-CD20 antibodies mediated cell death in vitro. PLoS One. 2014:9:e111113.

14. Weiner GJ. Rituximab: mechanism of action. Semin Hematol [Internet]. W.B. Saunders; 2010 [cited 2019 Jan 29];47:115-23. Available from: https://www. sciencedirect.com/science/article/pii/S0037196310000120?via\%3Dihub

15. Dotan E, Aggarwal C, Smith MR. Impact of rituximab (Rituxan) on the treatment of B-cell non-Hodgkin's lymphoma. P T. 2010;35:148-57.

16. Lee L, Crump M, Khor S, Hoch JS, Luo J, Bremner K, et al. Impact of rituximab on treatment outcomes of patients with diffuse large $b$-cell lymphoma: a population-based analysis. Br J Haematol. 2012;158:481-8.

17. Vose JM, Link BK, Grossbard ML, Czuczman M, Grillo-Lopez A, Gilman P, et al. Phase II study of rituximab in combination with CHOP chemotherapy in patients with previously untreated, aggressive non-Hodgkin's lymphoma. J Clin Oncol. 2001;19:389-97. 
18. Sehn LH, Donaldson J, Chhanabhai M, Fitzgerald C, Gill K, Klasa R, et al. Introduction of combined $\mathrm{CHOP}$ plus rituximab therapy dramatically improved outcome of diffuse large B-cell lymphoma in British Columbia. J Clin Oncol. 2005.

19. Pfreundschuh $M$, Trümper $L$, Österborg A, Pettengell $R$, Trneny M, Imrie K, et al. CHOP-like chemotherapy plus rituximab versus CHOP-like chemotherapy alone in young patients with good-prognosis diffuse large-Bcell lymphoma: a randomised controlled trial by the MabThera International Trial (MInT) Group. Lancet Oncol. 2006;7:379-91.

20. Coiffier B, Sarkozy C. Diffuse large B-cell lymphoma: R-CHOP failure-what to do? Hematology. 2016;1:366-78.

21. Amin AD, Peters TL, Li L, Rajan SS, Choudhari R, Puvvada SD, et al. Diffuse large B-cell lymphoma: can genomics improve treatment options for a curable cancer? Cold Spring Harb Mol Case Stud. 2017;3:a001719.

22. Sehn LH, Gascoyne RD. Diffuse large B-cell lymphoma: optimizing outcome in the context of clinical and biologic heterogeneity. Blood. 2015.

23. Eckschlager T, PIch J, Stiborova M, Hrabeta J. Histone deacetylase inhibitors as anticancer drugs. Int J Mol Sci. 2017;18.

24. Tang J, Wennerberg K, Aittokallio T. What is synergy? The Saariselkä agreement revisited. Front Pharmacol. 2015;6:1-5.

25. Cheung-Ong K, Giaever G, Nislow C. DNA-damaging agents in cancer chemotherapy: serendipity and chemical biology. Chem Biol. 2013;20:648-59.

26. Hoffman W, Lakkis FG, Chalasani G. B cells, antibodies, and more. Clin J Am Soc Nephrol. 2016;11:137-54

27. Johnson RD, Liu N, Jasin M. MammalianXRCC2 promotes the repair of DNA double-strand breaks by homologous recombination. Nature. 1999;401:397-9.

28. Ceccaldi R, Liu JC, Amunugama R, Hajdu I, Primack B, Petalcorin MI, et al. Homologous-recombination-deficient tumours are dependent on PolOmediated repair. Nature. 2015;518:258-62.

29. Ben-David U, Siranosian B, Ha G, Tang H, Oren Y, Hinohara K, et al. Genetic and transcriptional evolution alters cancer cell line drug response. Nature. 2018;560(7718):325-330

30. Bisserier M, Wajapeyee N. Mechanisms of resistance to ezh2 inhibitors in diffuse large b-cell lymphomas. Blood. 2018;19:962-73.

31. Mullighan CG, Zhang J, Kasper LH, Lerach S, Payne-Turner D, Phillips LA, et al. CREBBP mutations in relapsed acute lymphoblastic leukaemia. Nature. 2011;471:235-9.

32. Bitler BG, Wu S, Park PH, Hai Y, Aird KM, Wang Y, et al. ARID1A-mutated ovarian cancers depend on HDAC6 activity. Nat Cell Biol. 2017;19:962-73.

33. Caumanns JJ, Wisman GBA, Berns K, van der Zee AGJ, de Jong S. ARID1A mutant ovarian clear cell carcinoma: a clear target for synthetic lethal strategies. Biochim Biophys Acta - Rev Cancer. 1870;2018:176-84.

34. Bararia D, Heide M, Welner RS, Romashova T, Pastore A, Mathur R, et al. Disruptive ARID1A mutations in follicular lymphoma impair DNA repair efficiency and are associated with favorable outcome in patients receiving first-line immunochemotherapy. Blood. Am Soc Hematol. 2015;126:571.

35. Reddy A, Zhang J, Davis NS, Moffitt AB, Love CL, Waldrop A, et al. Genetic and functional drivers of diffuse large B cell lymphoma. Cell. 2017;171:481-94.

36. Anolik J, Looney RJ, Bottaro A, Sanz I, Young F. Down-regulation of CD20 on B cells upon CD40 activation. Eur J Immunol. 2003;33:2398-409.

37. Mariam H, Pierre Y, Adrian T, Gabriel T, Christian B, Anne B, et al. Membrane microdomain sphingolipids are required for anti-CD20-induced death of chronic lymphocytic leukemia B cells. Haematologica. Ferrata Storti Foundation. 2011;97:288-96 Available from: http://www.ncbi.nlm.nih.gov/ pmc/articles/PMC3269491/.

38. Whirl-Carrillo M, McDonagh EM, Hebert JM, Gong L, Sangkuhl K, Thorn CF, et al. Pharmacogenomics knowledge for personalized medicine. Clin Pharmacol Ther. 2012;92:414-7.

39. Till KJ, Coupland SE, Pettitt AR. Motility and trafficking in B-cell nonHodgkin's lymphoma (Review). Int J Oncol. 2014;45:5-12.

40. Peart MJ, Smyth GK, van Laar RK, Bowtell DD, Richon VM, Marks PA, et al. Identification and functional significance of genes regulated by structurally different histone deacetylase inhibitors. Proc Natl Acad Sci. 2005;102:3697-702.

41. Oronsky B, Oronsky N, Knox S, Fanger G, Scicinski J. Episensitization: therapeutic tumor resensitization by epigenetic agents: a review and reassessment. Anticancer Agents Med Chem. 2014;14:1121-7.

42. Batlevi CL, Kasamon Y, Bociek RG, Lee P, Gore L, Copeland A, et al. ENGAGE501: phase II study of entinostat (SNDX-275) in relapsed and refractory Hodgkin lymphoma. Haematologica. 2016;101:968-75.

43. Inc. E. Epizyme reports positive interim data from phase 2 trial for tazemetostat in relapsed or refractory follicular lymphoma and DLBCL patients [Internet]. Available from: https://globenewswire.com/newsrelease/2017/06/14/1018713/0/en/Epizyme-Reports-Positive-Interim-Datafrom-Phase-2-Trial-for-Tazemetostat-in-Relapsed-or-Refractory-FollicularLymphoma-and-DLBCL-Patients.html

44. Knutson SK, Kawano S, Minoshima Y, Warholic NM, Huang KC, Xiao Y, et al. Selective inhibition of EZH2 by EPZ-6438 leads to potent antitumor activity in EZH2-mutant non-Hodgkin lymphoma. Mol Cancer Ther. 2014;13:842-54.

45. Luzhna L, Golubov A, Inytskyy S, Chekhun VF, Kovalchuk O. Molecular mechanisms of radiation resistance in doxorubicin-resistant breast adenocarcinoma cells. Int J Oncol. 2013;42:1692-708.

46. Dobashi A. Molecular pathogenesis of diffuse large B-cell lymphoma. J Clin Exp Hematop. 2016:56:71-8.

47. Yap DB, Chu J, Berg T, Schapira M, Cheng SWG, Moradian A, et al. Somatic mutations at EZH2 Y641 act dominantly through a mechanism of selectively altered PRC2 catalytic activity, to increase H3K27 trimethylation. Blood. 2011; 117:2451-9.

48. Ocker M. Deacetylase inhibitors - focus on non-histone targets and effects. World J Biol Chem. 2010;1(5):55-61.

49. Falgreen S, Dybkær K, Young KH, Xu-Monette ZY, El-Galaly TC, Laursen MB, et al. Predicting response to multidrug regimens in cancer patients using cell line experiments and regularised regression models. BMC Cancer. 2015; 15:235.

50. Deeb SJ, D'Souza RCJ, Cox J, Schmidt-Supprian M, Mann M. Super-SILAC allows classification of diffuse large B-cell lymphoma subtypes by their protein expression profiles. Mol Cell Proteomics. 2012;11:77-89.

51. Leinonen $R$, Sugawara $H$, Shumway M. The sequence read archive. Nucleic Acids Res. 2011;39:D19-21.

\section{Publisher's Note}

Springer Nature remains neutral with regard to jurisdictional claims in published maps and institutional affiliations.

\section{Ready to submit your research? Choose BMC and benefit from:}

- fast, convenient online submission

- thorough peer review by experienced researchers in your field

- rapid publication on acceptance

- support for research data, including large and complex data types

- gold Open Access which fosters wider collaboration and increased citations

- maximum visibility for your research: over $100 \mathrm{M}$ website views per year

At BMC, research is always in progress.

Learn more biomedcentral.com/submissions 